doi:10.17659/01.2021.0034

Journal of Case Reports 2021;11(2):128-130

\title{
Non-Intubated Video-Assisted Thoracoscopy Using Epidural Anesthesia and Targeted Sedation in Mediastinal Mass
}

\author{
Isadora Megale Quadros, Marina Ayres Delgado, Camila Biazussi Damasceno, Paula Alves Pinheiro \\ Department of Anesthesia and Pain Medicine, Hospital das Clínicas da Universidade Federal de Minas Gerais, Belo Horizonte/ \\ Minas Gerais, Brazil.
}

\section{Corresponding Author:}

Dr Isadora Megale Quadros

Email: isadoramegaleq@gmail.com

This is an Open Access article distributed under the terms of the Creative Commons Attribution License (creativecommons.org/ licenses/by/3.0).

Received

Accepted

Published

October 23, 2020

April 13, 2021

June 20, 2021

\begin{abstract}
Background: Patients with mediastinal masses present unique challenges for anesthesiologists. If located in the anterior mediastinum they can cause obstruction of the airways and large vessels such as the pulmonary arteries and the superior vena cava (SVC). Case Report: During surgical procedures, acute cardiorespiratory collapse can be linked to general anesthesia due to tumor-related compression syndromes. Local anesthesia can be chosen in order to maintain spontaneous ventilation. Conclusion: This article aims to describe the case of a patient having a large anterior mediastinal mass with significant compressive symptoms who underwent thoracoscopy with biopsy.
\end{abstract}

Keywords: General Anesthesia, Epidural Anesthesia, Mediastinum, Mediastinal Diseases, Thoracoscopy.

\section{Introduction}

In patients with mediastinal masses, total occlusion of the airway and cardiovascular collapse are possible complications during the course of general anesthesia [1-2]. Many case reports have described fatal cardiopulmonary arrest during anesthesia for diagnostic or therapeutic surgical procedures in patients with mediastinal masses [3-4]. Most studies that assess the incidence of complications and identify patients at higher risk are carried out in pediatric populations [3]. The results cannot be extrapolated, in most cases, to adults because of the anatomic and physiologic differences among these groups of patients. The anesthetic management of patients with a mediastinal mass remains a clinical challenge. How it presents clinically varies depending where it is located and on tumor modifications in the mediastinum. It may range from complete lack of symptoms to severe cardiorespiratory responses. General anesthesia can be associated with cardiorespiratory decompensation and collapse, resulting in death due to tumor related compression syndromes $[3,5,6]$.

The purpose of this review is to present a case report of video-assisted thoracoscopy (VATS) in a patient with an anterior mediastinal mass, point out pathophysiologic considerations, factors that aid the anesthetic management and discuss the means of reducing the peri-operative risk.

\section{Case Report}

A 27-year-old male patient, sought health services having progressive dyspnea, aggravated by the supine position, dysphagia, and weight loss. Chest CT showed expansive and infiltrative tissue formation centered on the anterior mediastinum, with imprecise limits. It was in close contact with the pericardium, ascending aorta and right pulmonary artery. The caliber of the superior vena cava, trachea and main bronchi was reduced due to mass compression. 
He had been previously treated with prednisone $130 \mathrm{mg} /$ day, and then, admitted for mediastinal mass biopsy via right thoracotomy. The anesthesia plan was to maintain spontaneous breathing and adequate analgesia. Standard monitors were used with the patient in a semiseated position while an $18 \mathrm{G}$ peripheral venous access was placed in the left upper limb. A thoracic epidural was performed at T4-T5, using the loss of resistance technique, $20 \mathrm{ml}$ of $0.5 \%$ ropivacaíne was injected with $100 \mu \mathrm{g}$ of fentanyl. An epidural catheter was placed and maintained during the post-operative period. The patient was positioned in light left lateral decubitus for the surgical procedure while oxygen $\left(\mathrm{O}_{2}\right)$ via nasal catheter at $2 \mathrm{~L} / \mathrm{min}$ was maintained. The patient was sedated using dexmedetomidine $0.5 \mu \mathrm{g} / \mathrm{kg} / \mathrm{h}$, and ketamine $10 \mathrm{mg}$. The intra-operative course was stable, with no hemodynamic instabilities or difficulties in ventilation or oxygenation. After the procedure, the patient was transferred awake to the ICU and was using $\mathrm{O}_{2}$ through nasal catheter.

\section{Discussion}

The mediastinum contains vital organs in very close proximity: heart, great vessels, tracheobronchial tree, esophagus, nerves, thymus and lymphatics [3]. Traditionally, it has been divided into anterior, middle and posterior [4]. Typically, anterior mediastinal tumors cause more severe complications related to compression of the airways and vascular structures than others tumors. The most common malignancies that can present themselves as anterior mediastinal masses include: thymomas, Hodgkin lymphoma, T-non-Hodgkin lymphoma, germ cell tumors and mediastinal metastasis. The pre-operative management of patients with mediastinal mass begins with the assessment of the tumor's localization through clinical and radiological findings in order to establish the patient's risk classification [3]. Insufficient pre-operative preparation or incorrect diagnosis can quickly result in fatal outcomes.
The respiratory decompensation is mainly caused by mechanical compression of the trachea, bronchi or both by the mass. Even postural changes may cause a decompensation of the cardiorespiratory status. The supine position can reduce the transverse diameter of the thorax increasing the blood volume and consequently increasing the size of the vascularized mediastinal tumors and thereby, reducing the thoracic airway cross-sectional area with the increase of external compression pressure of the airways. Hemodynamic decompensation may occur if the heart or major vessels are compressed or infiltrated by mediastinal tumors. The effects of anterior mediastinal mass on the heart and great vessels can be related to external compression, invasive growth into the pericardium and vessel walls or presence of pleural or pericardial effusions. Malignant pericardial effusions can cause cardiac tamponade. Compression of the right cardiac chambers, superior vena cava, pulmonary arteries or pulmonary veins decrease the left ventricular preload and cardiac output [3-4]. The superior vena cava compression syndrome represents a lifethreatening situation, resulting in reduced venous drainage of the upper half of the body, decreased right ventricular filling pressure and cardiac output. Low intravascular pressure and thin vascular walls make the superior vena cava quite susceptible to external compression.

It is known that general anesthesia should be avoided in patients considered to be at high risk for peri-operative cardiorespiratory complications such as those with compressive symptoms [2]. Dyspnea, cough, hoarseness or syncope are signs of the severity of the disease especially when they occur upon moving the patient from a vertical to a horizontal position [6]. The presence of edema in the upper body and dilatation of the jugular are signs of deterioration of the superior vena cava. Liang et al. proposed that patients with pre-operative respiratory symptoms were more likely to experience airway compression and other 
complications during general anesthesia [1]. Our patient was treated before the procedure with corticoid pulse therapy in order to reduce the size of the mass and consequently, the symptoms and the risk of cardiorespiratory complication.

Thoracoscopy is an effective choice for diagnosis and treatment of mediastinal disorders. VATS permit biopsy procedures, good exposure of the mediastinum and great evaluation of the anatomic relationship of the tumor. For decades, intubated general anesthesia with one-lung ventilation was almost mandatory [1]. However, complications still may occur after intubation. If the anterior mediastinal mass sits at or below the level of the carina, inability to ventilate or oxygenate may persist, despite endotracheal intubation [4]. Data has shown that non-intubated VATS can be safely performed using epidural anesthesia and targeted sedation in order to maintain spontaneous respiration which avoids the residual effects of neuromuscular blocks and reduces the incidence of cardiorespiratory complications [7-8]. Spontaneous ventilatory activity prevents compression of the anterior mediastinum. Negative pressure ventilation normally preserves patency of intrathoracic airways whereas positive pressure worsens the airway compression by the tumor during the expiratory phase and decreases the venous return [5].

During the post-operative period, these patients should be observed and monitored as to the development of surgical complications as hemothorax, pneumothorax, and edema at the site of operation that can cause tracheal obstruction. In addition, pain after the intervention can lead to airway obstruction in the recovery room.

\section{Conclusion}

Alternatives to general anesthesia should be prioritized in high-risk patients for life-threatening respiratory complications. Non-intubated thoracoscopy surgery with epidural anesthesia is safe and can be an attractive choice for selected patients with mediastinal masses.

Contributors: IMQ and MAD were involved in planning and supervised the work, make substantial contributions to conception and design, drafted the article, wrote the paper with input from all authors; CBD and PAP gave final approval of the version to be submitted, revising it critically. IMQ will act as a study guarantor. All authors approved the final version of this manuscript and are responsible for all aspects of this study.

Funding: None; Competing interests: None stated.

\section{References}

1. Liang H, Liu J, Wu S, Zhang Y, Liu H, Yang H, et al. Nonintubated spontaneous ventilation offers better short-term outcome for mediastinal tumor surgery. Ann Thorac Surg. 2019;108(4):1045-1051.

2. Hartigan PM, Ng JM, Gill RR. Anesthesia in a patient with a large mediastinal mass. New England Journal of Medicine. 2018;379:587-588.

3. Brenn BR, Hughes AK. The anesthetic management of anterior mediastinal masses in children: A Review. International Anesthesiology Clinics. 2019;57:E24-41.

4. Pearson JK, Tan GM. Pediatric Anterior Mediastinal Mass. Vol. 19, Seminars in Cardiothoracic and Vascular Anesthesia. SAGE Publications Inc.; 2015. pp. 248-254.

5. Malik R, Mullassery D, Kleine-Brueggeney M, Atra A, Gour A, Sunderland R, et al. Anterior mediastinal masses - A multidisciplinary pathway for safe diagnostic procedures. J Pediatr Surg. 2019;54(2):251-254.

6. Acker SN, Linton J, Tan GM, Garrington TP, Bruny J, Hilden JM, et al. A multidisciplinary approach to the management of anterior mediastinal masses in children. J Pediatr Surg. 2015;50(5):875-878.

7. Cui F, Liu J, Li S, Yin W, Xin X, Shao W, et al. Tubeless video-assisted thoracoscopic surgery (VATS) under nonintubated, intravenous anesthesia with spontaneous ventilation and no placement of chest tube postoperatively. J Thorac Dis. 2016;8(8):2226-2232.

8. Liu J, Cui F, Li S, Chen H, Shao W, Liang L, et al. Nonintubated video assisted thoracoscopic surgery under epidural anesthesia compared with conventional anesthetic option: A randomized control study. Surg Innov. 2015;22(2):123-130. 